\title{
VLBI Observations of SN 1979C and SN 1986J
}

\author{
N. Bartel and M.F. Bietenholz \\ York University, Toronto, Canada, \\ bartel@yorku.ca
}

Summary. We summarize our results on multi-epoch VLBI observations of SN $1979 \mathrm{C}$ in the galaxy M100 in Virgo, and of SN 1986 in the galaxy NGC 891. From $t=3.7$ to $22 \mathrm{yr}$ after the explosion, SN $1979 \mathrm{C}$ expands $\propto t^{m}$, almost freely, with $m=0.95 \pm 0.03$. For a total kinetic energy of $3 \times 10^{51} \mathrm{erg}$, the expansion result requires a mass-loss to wind-velocity ratio for the progenitor of only $1 \times$ $10^{-5} \mathrm{M}_{\odot} \mathrm{yr}^{-1}$ per $10 \mathrm{~km} \mathrm{~s}^{-1}$, an order of magnitude smaller than estimated from radio light-curve fitting. We show a first image with slightly discernible structure of the supernova. For SN 1986J we present five images from 1987 to 2002 and show our result on moderately to strongly decelerated expansion with $m=0.71 \pm 0.11$. We comment on our result of an inversion of the radio spectrum in terms of the emergence of a possible pulsar nebula.

The supernovae, SN 1979C [3] and SN 1986J [4] are, after SN 1993J, the next most comprehensively monitored radio supernovae with VLBI to date.

SN 1979C: In Fig. 1 we show a radio image of M100 with SN 1979C located at the southern edge of a spiral arm. We also show an image of SN $1979 \mathrm{C}$ at $t=22 \mathrm{yr}$ after the explosion revealing for the first time structure, namely along the minor axis of the beam. SN $1979 \mathrm{C}$ was the first supernova whose radius and expansion could be determined with VLBI [2]. Our latest results (Fig. 2) show an expansion of the size $\propto t^{m}$ which is, with $m=$ $0.95 \pm 0.03$, almost consistent with being free. This result is in conflict with a recent report of strong deceleration starting at $t=6 \pm 2 \mathrm{yr}$ [5]. For our solution for $m$, the ratio of the mass of the shocked ejecta to that of the swept up wind, $M_{\text {shock-ej }} / M_{\mathrm{sw}}=7_{-3}^{+13}$ for a density profile of the circumstellar medium (CSM) of $\rho_{C S M} \propto r^{-2}$. With the mass-loss to wind-velocity ratio taken from radio light-curve fits of $\dot{M}_{w} / w=1.6 \times 10^{-4} \mathrm{M}_{\odot} \mathrm{yr}^{-1}$ per $10 \mathrm{~km} \mathrm{~s}^{-1}$ [6], we get $M_{\text {shock-ej }}=25_{-10}^{+50} \mathrm{M}_{\odot}$ at $t=22 \mathrm{yr}$. This value is excessively large. In fact, with the velocity derived from the expansion curve, we get for the kinetic energy of the shells of the shocked ejecta and the shocked CSM, $E_{\text {kin,shock }}=1.5_{-0.5}^{+3} \times 10^{52} \mathrm{erg}$. The total kinetic energy, including the kinetic energy of the unshocked ejecta, is even larger, about a factor of three.

With the explosion energy for a type II supernova of $\sim 3 \times 10^{51} \mathrm{erg}$, we can assume a typical value for $E_{\text {kin,shock }}$ of $\sim 1 \times 10^{51} \mathrm{erg}$. We therefore think that $\dot{M}_{w} / w \sim 1 \times 10^{-5} \mathrm{M}_{\odot} \mathrm{yr}^{-1}$ per $10 \mathrm{~km} \mathrm{~s}^{-1}$. This value is about an order of magnitude smaller than that estimated from radio light-curve fitting. Values 


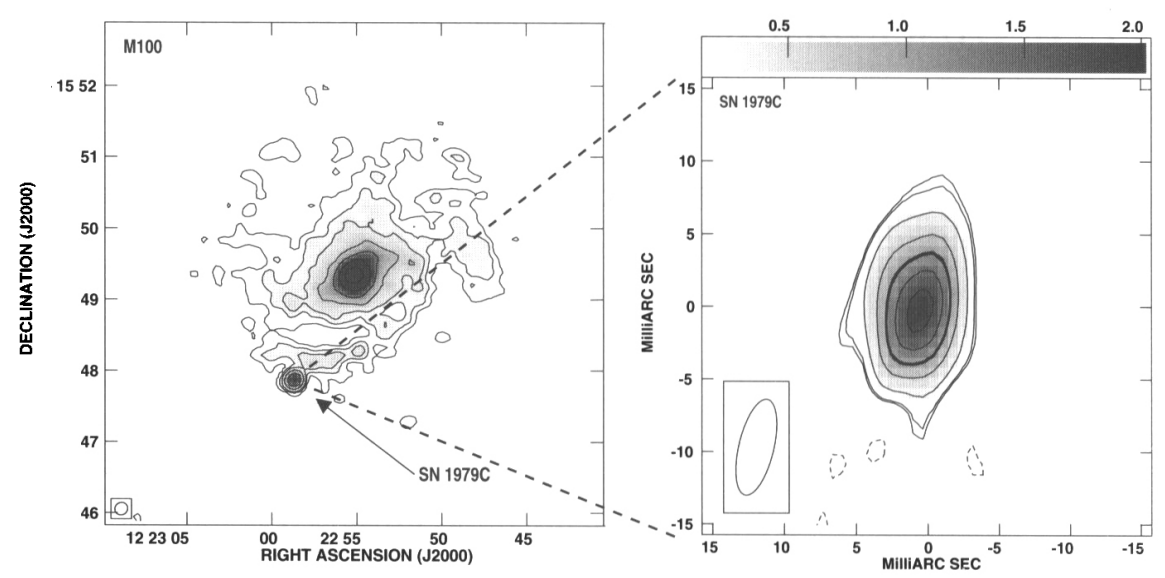

Fig. 1. Left panel: VLA image of the spiral galaxy M100 in the Virgo cluster with SN $1979 \mathrm{C}$ in it, at $1.7 \mathrm{GHz}$ on 1996 March 15 . Right panel: VLBI image of SN $1979 \mathrm{C}$ at $1.7 \mathrm{GHz}$ on 2001 February 24. The beam (FWHM, 6.8 mas $\times 2.4$ mas at a p.a. of $-14 \mathrm{deg}$ ) is shown at lower left. The grey scale is labeled in $\mathrm{mJy}_{\mathrm{beam}}{ }^{-1}$.

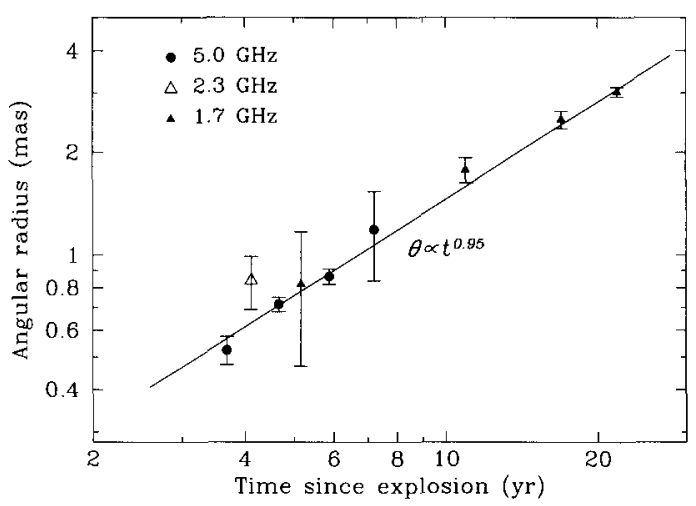

Fig. 2. The expansion of the angular radius, $\theta$, of a uniform sphere model fit to the $u-v$ data. The solid line gives the weighted least-squares power-law fit.

of $\dot{M}_{w} / w$ obtained from radio light-curve fitting should therefore be treated with caution.

The Expanding Shock Front Method (ESM) of combining the transverse radio expansion velocities with the radial optical velocities gives direct distance estimates, with standard errors, of $16.5 \pm 2.5$ to $19.8 \pm 3.0 \mathrm{Mpc}$ depending on whether the supernova has a bright center or is a shell without such a center. 


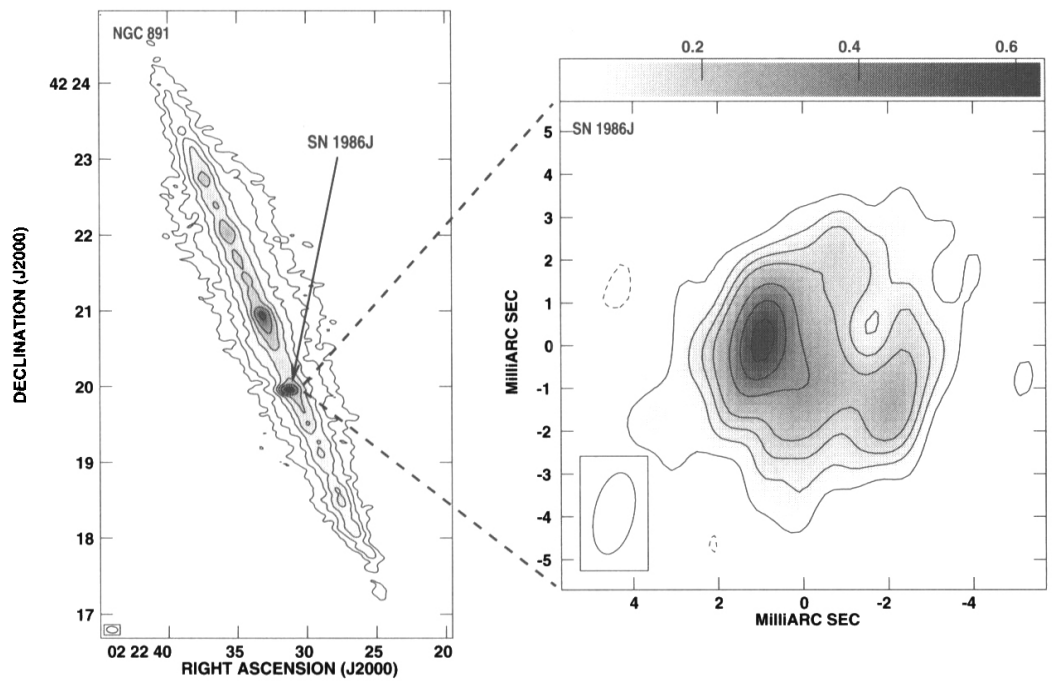

Fig. 3. Left panel: VLA image of NGC 891 with $\mathrm{SN} 1986 \mathrm{~J}$ in it, at $5 \mathrm{GHz}$ on 1999 February 22. Right panel: VLBI image of SN $1986 \mathrm{~J}$ at $5.0 \mathrm{GHz}$ at epoch 2002 November 11. The beam is shown at lower left. The grey scale is labeled in mJy beam ${ }^{-1}$. In addition, contours are drawn at $-7.4,7.4,10,15,20,25,30,40$, 50,70 , and $90 \%$ of the peak brightness of $0.57 \mathrm{mJy}_{\text {beam }}{ }^{-1}$.

SN 1986J: In Fig. 3 we show a radio image of the edge-on galaxy NGC 891 with SN 1986J located prominently in the southwestern part of it. We also show the latest image of SN 1986J at $t=20 \mathrm{yr}$, which reveals the complex structure of the supernova.

The full sequence of one model and four subsequent images is displayed in Fig. 4. The source structure is rather complex. It may be a composite with large brightness modulations across the source and with protrusions. Apart from an early high-resolution image [1], Fig. 5 gives the best indication so far that some components of shell structure are present.

The radio spectrum (Fig. 6, left panel) is inverted above $\sim 10 \mathrm{GHz}$ and may indicate the emergence of a pulsar nebula. Perhaps component $\mathrm{C} 1{ }^{*}$ in Fig. 5 is a candidate for such a nebula. The expansion (Fig. 6, right panel) is moderately to strongly decelerated with $m=0.71 \pm 0.11$. Note, that this result is in conflict with that reported by [7]. 

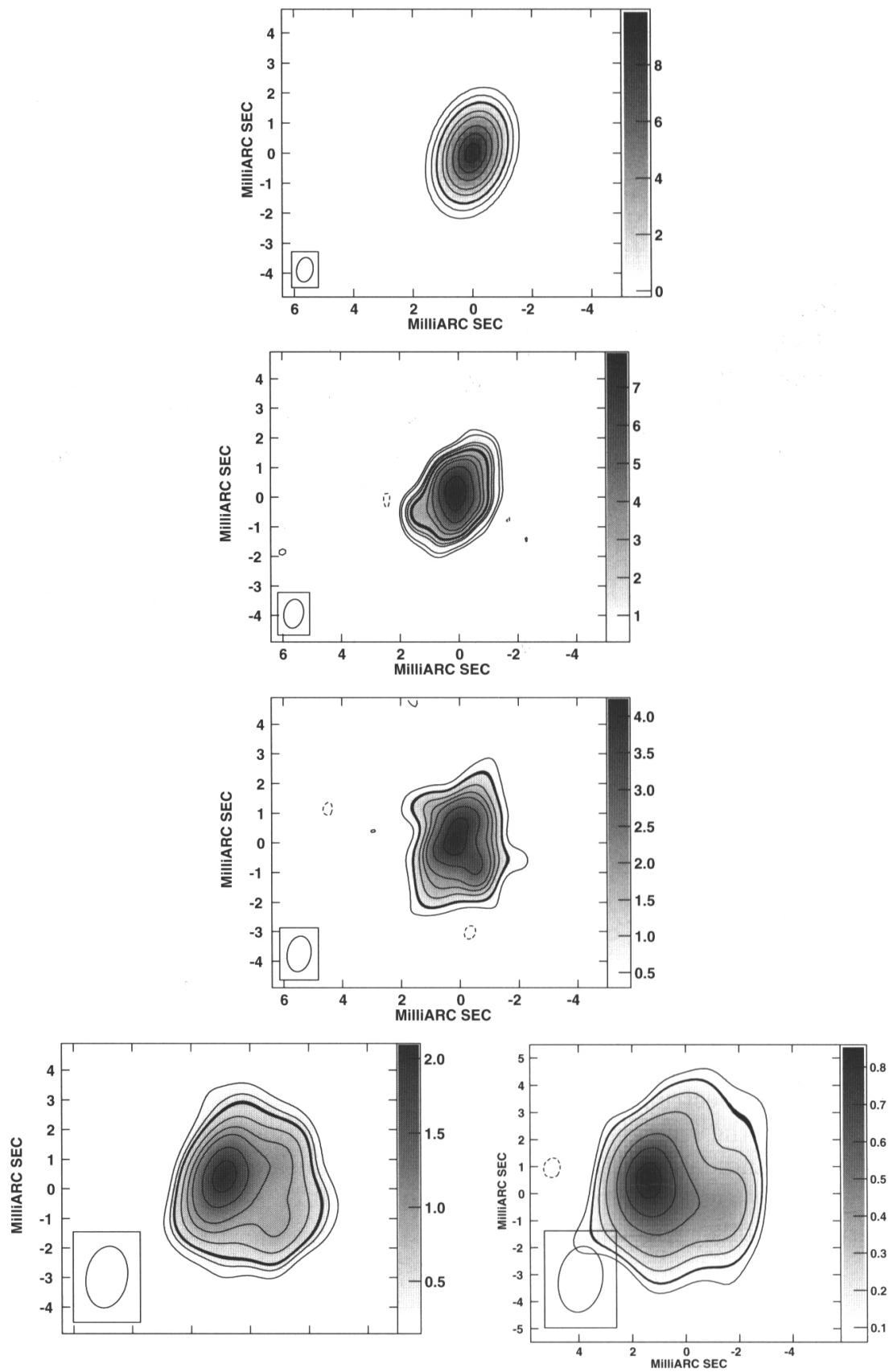

Fig. 4. One model (first panel, $1987 \mathrm{Feb}, 10.7 \mathrm{GHz}$ ) and four images (1988 Sep., 8.4 GHz; 1990 Jul., 8.4 GHz; 1999 Feb., $5.0 \mathrm{GHz} ; 2002$, Nov., $5.0 \mathrm{GHz}$ ) of SN 1986J. The heavier contour is that which contains $90 \%$ of the flux density. The brightness is given on the right margin in each panel in $\mathrm{mJy}_{\mathrm{beam}}{ }^{-1}$. The dynamically evolving beam is given in the lower left. 


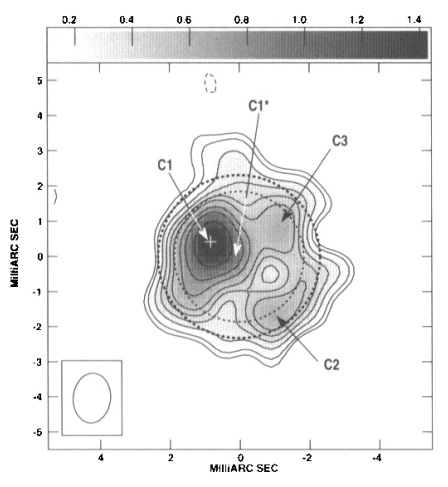

Fig. 5. VLBI image of SN 1986J at $5.0 \mathrm{GHz}$ at epoch 1999 February 22. Contours are drawn at $-7.4,7.4,10,15,20,25,30,40,50,70$, and $90 \%$ of the peak brightness of $1.64 \mathrm{mJy} \mathrm{beam}^{-1}$. The dotted circles indicate the inner and outer radii of a spherical shell fit to the $u-v$ data. Note, that the brightest portion of a projected shell of uniform volume emissivity is along the inner circle. See [7] for an independent analysis of our observations.
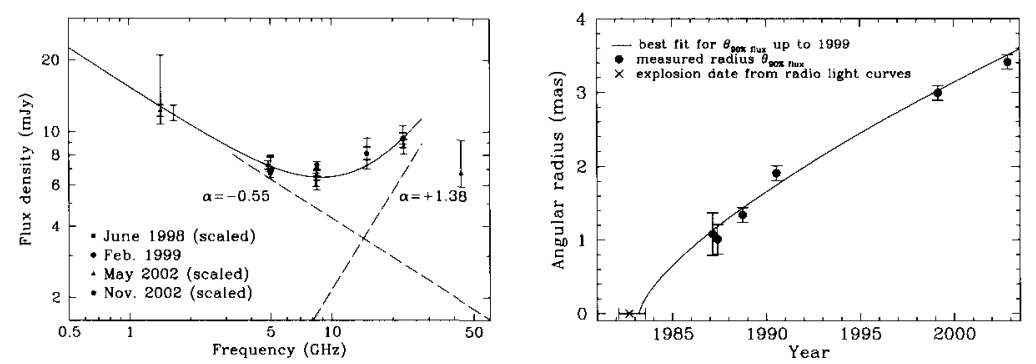

Fig. 6. Left panel: The radio spectrum of SN 1986J. The measurements in 1998 and 2002 were scaled to those of 1999 . The dashed lines show the decomposition into two spectral power-law components. Right panel: An expansion power-law fit to all but the last measurements (from $90 \%$ flux density contours).

\section{References}

1. N. Bartel, M.P. Rupen, I.I. Shapiro, R.A. Preston, A. Rius: Nature 350, $212(1991)$

2. N. Bartel, A.E.E. Rogers, I.I. Shapiro, M.V. Gorenstein, C.R. Gwinn, J.M. Marcaide, and K.W. Weiler: Nature 318, 25 (1985)

3. N. Bartel and M.F. Bietenholz: Astrophys. J. 591, 301 (2003)

4. M.F. Bietenholz, N. Bartel, M.P. Rupen: Astrophys. J. 581, 1132 (2002)

5. J.M. Marcaide et al. : Astron. Astrophys. 384, 408 (2002)

6. M.J. Montes, K.W. Weiler, S.D. Van Dyk, N.Panagia, C.K. Lacey, R.A. Sramek, R. Park: Astrophys. J. 532, 1124 (2000)

7. M.A. Pérez-Torres, A. Alberdi, J.M. Marcaide, J.C. Guirado, L. Lara, F. Mantovani, E. Ros, K.W. Weiler: Mon. Not. R. Astron. Soc. 335, L23 (2002) 